\title{
ON THE PAIR CORRELATIONS OF POWERS OF REAL NUMBERS
}

\author{
CHRISTOPH AISTLEITNER AND SIMON BAKER
}

\begin{abstract}
A classical theorem of Koksma states that for Lebesgue almost every $x>1$ the sequence $\left(x^{n}\right)_{n=1}^{\infty}$ is uniformly distributed modulo one. In the present paper we extend Koksma's theorem to the pair correlation setting. More precisely, we show that for Lebesgue almost every $x>1$ the pair correlations of the fractional parts of $\left(x^{n}\right)_{n=1}^{\infty}$ are asymptotically Poissonian. The proof is based on a martingale approximation method.
\end{abstract}

\section{INTRODUCTION}

Let $\left(x_{n}\right)_{n=1}^{\infty}$ be a sequence of real numbers in the unit interval. We say that $\left(x_{n}\right)$ has Poissonian pair correlations if for every $s>0$ :

$$
\lim _{N \rightarrow \infty} \frac{\#\left\{1 \leq m \neq n \leq N:\left\|x_{n}-x_{m}\right\| \leq \frac{s}{N}\right\}}{N}=2 s .
$$

Here and throughout $\|\cdot\|$ denotes the distance to the nearest integer, and $\{\cdot\}$ denotes the fractional part. It can be shown that if $\left(y_{n}\right)$ is a sequence whose entries are chosen from $[0,1]$ independently and with respect to the uniform probability measure, then almost surely $\left(y_{n}\right)$ has Poissonian pair correlations. Accordingly, one can view the property of having Poissonian pair correlations as an indication of the "random" behaviour of a sequence modulo one.

Part of the motivation behind studying whether a sequence has Poissonian pair correlations comes from a connection with quantum physics. The Berry-Tabor conjecture states that the discrete energy spectrum of a quantum system has Poissonian pair correlations except for in certain degenerate cases. See 23 for the background in theoretical physics. For some special quantum systems it can be shown that there exists $\alpha \in \mathbb{R}$, and a sequence of positive integers $\left(a_{n}\right)$, such that the local distribution properties of the discrete energy spectrum of the system agree with those of the sequence $\left(\left\{a_{n} \alpha\right\}\right)$. This connection lead to several important works of Rudnick, Sarnak, and Zaharescu [28, 29, 30]. Interestingly

2010 Mathematics Subject Classification. 11K06, 11K60.

Key words and phrases. Poissonian pair correlations, powers of real numbers, Koksma's theorem, pseudorandomness, metric number theory. 
the sequence $(n \alpha)$ does not have Poissonian pair correlations for any $\alpha \in \mathbb{R}$. This can be viewed as a consequence of the three gap theorem. See [22] for details.

From a number-theoretic perspective it is an interesting and challenging problem to determine whether a sequence $\left(x_{n}\right)$ has Poissonian pair correlations or not. However, there exist only very few positive results in this direction. For example, it is conjectured that $\left(\left\{n^{2} \alpha\right\}\right)$ has Poissonian pair correlations, provided that $\alpha$ cannot be approximated very well by rationals. There are partial results in this direction (see [17, 32]), but it seems that the full conjecture is wide out of reach. In contrast, the situation is relatively wellunderstood from a metric point of view. Let $\left(a_{n}\right)$ be a sequence of distinct positive integers. Then for Lebesgue almost every $\alpha$ the sequence $\left(\left\{a_{n} \alpha\right\}\right)$ has Poissonian pair correlations, unless the so-called additive energy of the sequence $\left(a_{n}\right)$ is exceptionally large. See [3] and [10].

In this paper we are interested in the distribution of fractional parts of sequences of the form $\left(x^{n}\right)$, for $x>1$. The study of this family of sequences dates back to work of Hardy [16] who proved that if $x$ is an algebraic number, then $\lim _{n \rightarrow \infty}\left\|x^{n}\right\|=0$ if and only if $x$ is a Pisot number. This result was obtained independently by Pisot in [26]. Pisot had proved in an earlier work [27] that there are at most countably many $x>1$ satisfying $\lim _{n \rightarrow \infty}\left\|x^{n}\right\|=0$. Determining whether there exists transcendental $x>1$ satisfying $\lim _{n \rightarrow \infty}\left\|x^{n}\right\|=0$ is still an important open problem. The first metric results on the distribution of the sequence $\left(x^{n}\right)$ were due to Koksma. In [19] he proved that for Lebesgue almost every $x>1$ the sequence $\left(x^{n}\right)$ is uniformly distributed modulo one. A version of this theorem for selfsimilar measures was recently established by the second author in [4]. In [20], Koksma proved that for any sequence of real numbers $\left(y_{n}\right)$, under suitable monotonicity conditions on the sequence $\left(\epsilon_{n}\right)$, the set

$$
\left\{x>1:\left\|x^{n}-y_{n}\right\| \leq \epsilon_{n} \text { for infinitely many } n \in \mathbb{N}\right\}
$$

has zero or full Lebesgue measure depending on whether $\sum_{n=1}^{\infty} \epsilon_{n}$ diverged or converged. For some more recent work on the distribution of the sequence $\left(x^{n}\right)$ we refer the reader to [1, 4, 5, 6, 12, 13, 14, 18], as well as to [11, Chapters 2 and 3], and the references therein.

In [5] the second author asked the question:

Question 1.1. Is it true that for Lebesgue almost every $x>1$ the sequence $\left(\left\{x^{n}\right\}\right)_{n=1}^{\infty}$ has Poissonian pair correlations?

The second author was unable to answer this question. Instead he proved via a second moment argument that if $\left(d_{n}\right)$ is a sequence of integers that satisfies a certain growth condition, then for Lebesgue almost every $x>1$ the sequence $\left(\left\{x^{d_{n}}\right\}\right)$ has Poissonian pair 
correlations. For example one can take $\left(d_{n}\right)$ to be $\left(n^{k}\right)_{n=1}^{\infty}$ for any $k \geq 2$. In this paper we obtain a positive answer to the question above. In particular we prove the following theorem.

Theorem 1.2. For Lebesgue almost every $x>1$ the sequence $\left(\left\{x^{n}\right\}\right)_{n=1}^{\infty}$ has Poissonian pair correlations.

It is known that having Poissonian pair correlations is a stronger property than being equidistributed; see for example [2, 15]. Thus our theorem is indeed an extension of Koksma's theorem. A simple adaptation of our proof of Theorem 1.2 shows that for any fixed $\xi \neq 0$ the sequence $\left(\left\{\xi x^{n}\right\}\right)_{n=1}^{\infty}$ has Poissonian pair correlations for Lebesgue almost every $x>1$. For simplicity we restrict our attention to the case $\xi=1$.

We give a brief heuristic for the theorem, and outline the strategy of proof. Let us first consider a so-called lacunary sequence of integers $\left(a_{n}\right)$, that is, a sequence of at least exponential growth. It is a classical observation that in this case the functions $\left(\left\{a_{n} \alpha\right\}\right)$ exhibit properties which are typical for sequences of independent, identically distributed (i.i.d.) random variables. In the context of pair correlations, Rudnick and Zaharescu [31] proved that for a lacunary sequence $\left(a_{n}\right)$, the pair correlations of $\left(\left\{a_{n} \alpha\right\}\right)$ are Poissonian for Lebesgue almost every $\alpha$. Actually, their results go far beyond pair correlations: they could show that for generic $\alpha$ also the triple correlations and all other higher correlations coincide with the Poissonian model, which implies that the distribution of the level spacings (nearest-neighbour spacings) is asymptotically exponential (exactly as the distribution of spacings in the Poisson process - this is the source of the term "Poissonian"). The reason for this "pseudo-random" behaviour of the sequence $\left(\left\{a_{n} \alpha\right\}\right)$ is the oscillatory nature with quickly growing frequencies of these functions (which is exploited in the proof in [31] by reducing the problem to the counting of the number of solutions of certain Diophantine equations). In a similar way, the sequence of functions $\left(\left\{x^{n}\right\}\right)$ exhibits oscillatory and "pseudo-random" behaviour; Donald Knuth mentions such sequences in his celebrated The art of computer programming as examples of sequences exhibiting a high degree of pseudorandomness, and stated a conjecture on their statistical properties which was solved by Niederreiter and Tichy [24]. From a technical perspective, sequences of the form $\left(\left\{x^{n}\right\}\right)$ are significantly more difficult to handle than lacunary sequences, since they are lacking the simple "homogeneous" nature of lacunary sequences, which by orthogonality of the trigonometric system allows the reduction of moment estimates to a simple counting of solutions of Diophantine equations. To overcome these problems, in the present paper we set up a martingale approximation machinery to prove Theorem 1.2, which makes direct 
use of the oscillatory nature of the sequence $\left(\left\{x^{n}\right\}\right)$. This martingale method was introduced in metric number theory for problems concerning lacunary trigonometric sequences independently by Berkes [7, 8] and Philipp and Stout [25]. In the context of pair correlation problems (for the case of lacunary sequences), it was used by Berkes, Philipp and Tichy in [9]. Roughly speaking, the functional principle of the method is the observation that when we have two oscillating functions, where the frequency of oscillation of the second function is much higher than that of the first, then we can closely approximate the first function by a step function such that the second function still quickly oscillates on the intervals where the step function is constant - or, in probabilistic language, the conditional expectation of the second function is essentially independent of the sigma-field under which the discretization of the first function is measurable. This allows to approximate the whole structure by a martingale, and it is known from probability theory that in many respects martingales show the same distributional behaviour as sums of independent random variables. The martingale approximation is technically involved, which prevented us from addressing the case of triple or higher correlations. However, we believe that these correlations should also follow the Poissonian model, for Lebesgue almost every $\alpha$.

Conjecture 1.3. For Lebesgue almost every $x>1$ the triple correlations of the sequence $\left(\left\{x^{n}\right\}\right)_{n=1}^{\infty}$ coincide with the Poissonian model. The same is true for all higher correlations, as well as for the level spacings.

Theorem 1.2 is equivalent to the following statement: for Lebesgue almost every $x>1$, for all $s>0$ we have

$$
\lim _{N \rightarrow \infty} \frac{\sum_{1 \leq m \neq n \leq N} \chi_{\left[\frac{-s}{N}, \frac{s}{N}\right]}\left(x^{n}-x^{m}\right)}{N}=2 s .
$$

Here, and in the sequel, $\chi_{\left[\frac{-s}{N}, \frac{s}{N}\right]}$ denotes the indicator function on the set $\mathbb{Z}+\left[\frac{-s}{N}, \frac{s}{N}\right]$. Instead of studying the function $\chi_{\left[\frac{-s}{N}, \frac{s}{N}\right]}$ directly it is more convenient to study a suitable smooth approximation. Most of this paper will be concerned with proving the following theorem which considers such approximations.

Theorem 1.4. Let $s>0$ be fixed. Suppose $\left(F_{N}\right)_{N=1}^{\infty}$ is a sequence of differentiable functions satisfying:

(1) $F_{N}(x+1)=F_{N}(x)$ for all $x \in \mathbb{R}$ and $N \in \mathbb{N}$.

(2) $F_{N}(x)=F_{N}(-x)$ for all $x \in \mathbb{R}$ and $N \in \mathbb{N}$.

$$
\int_{0}^{1} F_{N} d x=\frac{2 s}{N}+\mathcal{O}\left(\frac{1}{N^{2}}\right) .
$$


(4) $0 \leq F_{N}(x) \leq 1$ for all $x \in \mathbb{R}$ and $N \in \mathbb{N}$.

(5) $\left\|F_{N}^{\prime}\right\|_{\infty}=\mathcal{O}\left(N^{2}\right)$.

(6) $\operatorname{supp}\left(F_{N}\right) \subseteq \mathbb{Z}+\left[\frac{-2 s}{N}, \frac{2 s}{N}\right]$.

Then Lebesgue almost every $x>1$ satisfies

$$
\lim _{N \rightarrow \infty} \frac{\sum_{1 \leq m \neq n \leq N^{20}} F_{N^{20}}\left(x^{n}-x^{m}\right)}{N^{20}}=2 s .
$$

Once Theorem 1.4 is established Theorem 1.2 will follow almost immediately.

1.1. Structure of the paper and notational conventions. In Section 2 we prove Theorem 1.4. In Section 3 we prove Theorem 1.2. Throughout this paper we will use the standard big $\mathcal{O}$ notation. That is we say $X=\mathcal{O}(Y)$ if there exists $C>0$ such that $|X| \leq C Y$. We will use $\mathcal{L}(\cdot)$ to denote the Lebesgue measure.

\section{Proof of Theorem 1.4}

We now proceed with our proof of Theorem 1.4. Throughout this section the parameter $s>0$ will be fixed and $\left(F_{N}\right)_{N=1}^{\infty}$ will be a sequence of functions satisfying the hypothesis of Theorem 1.4. We also choose a number $A>1$ arbitrarily, and keep its value fixed throughout this section. Since $A$ and $s$ are considered to be fixed, in our proof we will suppress the dependence of the implied $\mathcal{O}$ constants on $A$ and $s$.

For each $N \in \mathbb{N}$ let

$$
G_{N}:=F_{N}-\int_{0}^{1} F_{N} d x .
$$

It will be technically more convenient to study the typical behaviour of

$$
\sum_{1 \leq m \neq n \leq N} G_{N}\left(x^{n}-x^{m}\right)
$$

rather than

$$
\sum_{1 \leq m \neq n \leq N} F_{N}\left(x^{n}-x^{m}\right)
$$

By our underlying assumptions we know that $F_{N}(x)=F_{N}(-x)$ for all $x \in \mathbb{R}$. Therefore $G_{N}(x)=G_{N}(-x)$ for all $x \in \mathbb{R}$. This implies

$$
\sum_{1 \leq m \neq n \leq N} G_{N}\left(x^{n}-x^{m}\right)=2 \sum_{1 \leq m<n \leq N} G_{N}\left(x^{n}-x^{m}\right) .
$$

As such, in our analysis we may always assume $m<n$.

We now partition the set $\{1, \ldots, N\}$ into blocks which describe the magnitude of the parameter $n$ on an appropriate scale. Suppose $N$ is some large number. As the conclusion of Theorem 1.4 indicates, we will only need to consider values of $N$ which are the 20th 
power of an integer. Thus we can assume throughout this section (for simplicity of writing) that $N^{1 / 10}$ is an integer, and that $N^{9 / 10}$ is an integer as well.

For each $1 \leq k \leq N^{9 / 10}$ let

$$
\Delta_{k, N}:=\left\{(k-1) N^{1 / 10}+1, \ldots, k N^{1 / 10}\right\} .
$$

Note that obviously we have

$$
\# \Delta_{k, N}=N^{1 / 10} \quad \text { and } \quad \bigcup_{k=1}^{N^{9 / 10}} \Delta_{k, N}=\{1, \ldots, N\} .
$$

For each $1 \leq k \leq N^{9 / 10}$ let us define

$$
Y_{k, N}(x):=\sum_{n \in \Delta_{k, N}} \sum_{1 \leq m<n} G_{N}\left(x^{n}-x^{m}\right) .
$$

It follows from (2.1) that

$$
\sum_{1 \leq m \neq n \leq N} G_{N}\left(x^{n}-x^{m}\right)=2\left(\sum_{\substack{1 \leq k \leq N^{9 / 10}, k \text { is odd }}} Y_{k, N}(x)+\sum_{\substack{1 \leq k \leq N^{9 / 10}, k \text { is even }}} Y_{k, N}(x)\right) .
$$

Conditioning on the parity of $k$ within (2.2) will play an important part in our proof of Theorem 1.4. By adopting this approach we can obtain stronger quantitative information on the independence between the functions $Y_{k, N}$ and $Y_{j, N}$.

The key to proving Theorem 1.4 will be following proposition.

Proposition 2.1. Let $s>0$ and suppose $\left(F_{N}\right)_{N=1}^{\infty}$ is a sequence of functions satisfying the hypothesis of Theorem 1.4. Then

$$
\int_{A}^{A+1}\left(\sum_{\substack{1 \leq k \leq N^{9 / 10}, k \text { is odd }}} Y_{k, N}\right)^{2} d x=\mathcal{O}\left(N^{11 / 10}\right) .
$$

The same result holds if the summation is extended over all even (rather than all odd) values of $k$ in the specified range.

Expanding the bracket within Proposition 2.1 we obtain

$$
\int_{A}^{A+1}\left(\sum_{\substack{1 \leq k \leq N^{9 / 10}, k \text { is odd }}} Y_{k, N}\right)^{2} d x
$$




$$
=\int_{A}^{A+1} 2 \sum_{\substack{1 \leq j<k \leq N^{9 / 10}, j, k \text { are odd }}} Y_{j, N} \cdot Y_{k, N} d x+\int_{A}^{A+1} \sum_{\substack{1 \leq k \leq N^{9 / 10}, k \text { is odd }}} Y_{k, N}^{2} d x .
$$

A similar equation holds in the even case. Proposition 2.1 will be implied by the following two lemmas.

Lemma 2.2. Suppose $k-j \geq 2$. Then

$$
\int_{A}^{A+1} Y_{j, N} \cdot Y_{k, N} d x=\mathcal{O}\left(\frac{\log N}{N^{18 / 10}}\right) .
$$

Lemma 2.3. For all $1 \leq k \leq N^{9 / 10}$ we have

$$
\int_{A}^{A+1} Y_{k, N}^{2} d x=\mathcal{O}\left(N^{2 / 10}\right)
$$

The first summation on the right hand side of (2.3) consists of $\mathcal{O}\left(N^{18 / 10}\right)$ terms, and the second summation consists of $\mathcal{O}\left(N^{9 / 10}\right)$ terms. Therefore, applying the bounds provided by Lemma 2.2 to the first summation, and the bounds provided by Lemma 2.3 to the second summation, we may conclude that Proposition 2.1 holds in the odd case. The even case follows by similar reasoning. Therefore to prove Proposition 2.1 it suffices to prove Lemma 2.2 and Lemma 2.3. This we do in the next two sections.

2.1. Proof of Lemma 2.2. To prove Lemma 2.2 we will introduce a collection of stepfunctions $Z_{k, N}$ that provide good approximations to $Y_{k, N}$. For this collection of functions it will be easier to prove that for $k-j \geq 2$ the integral $\int_{A}^{A+1} Z_{j, N} \cdot Z_{k, N} d x$ is small. Before we can define $Z_{k, N}$ we need to introduce the following $\sigma$-algebra.

Given $k \in \mathbb{N}$ and $x \in[A, A+1)$, we define the quantity $\mu_{k}(x)$ to be the unique integer solution to the inequalities:

$$
2^{\mu_{k}(x)} \leq x^{(k+1 / 2) N^{1 / 10}}<2^{\mu_{k}(x)+1} .
$$

Now let us fix $k \in \mathbb{N}$. Let $z_{0, k}=A$ and define $z_{1, k}=A+2^{-\mu_{k}(A)}$. Suppose $z_{0, k}, \ldots, z_{j, k}$ have been constructed and $z_{j, k}<A+1$, we define $z_{j+1, k}$ to be $z_{j+1, k}=z_{j, k}+2^{-\mu_{k}\left(z_{j, k}\right)}$. We stop at $N_{k}$ when $z_{N_{k}, k}=A+1$. Such an $N_{k}$ must exist because $\mu_{k}(x)$ is bounded from above, increasing with $x$, and because at each step in our construction we are adding a negative power of 2 . We define $\mathcal{F}_{k}$ to be the $\sigma$-algebra generated by the intervals

$$
\left\{\left[z_{i, k}, z_{i+1, k}\right): 0 \leq i<N_{k}\right\} .
$$

Note that $\mu_{k}(x) \leq \mu_{k+1}(x)$ for all $x \in[A, A+1)$. Using this property, and the fact that each $\mathcal{F}_{k}$ is generated by a collection of intervals whose length is some negative power of 2 , we can conclude that $\mathcal{F}_{j}$ is a sub $\sigma$-algebra of $\mathcal{F}_{k}$ for all $j<k$. 
For each $1 \leq k \leq N^{9 / 10}$ we define $Z_{k, N}:[A, A+1) \rightarrow \mathbb{R}$ as follows:

$$
Z_{k, N}(x)=\frac{1}{\mathcal{L}\left(\left[z_{i, k}, z_{i, k+1}\right)\right)} \int_{z_{i, k}}^{z_{i, k+1}} Y_{k, N} d x \quad \text { if } x \in\left[z_{i, k}, z_{i, k+1}\right) \text { for some } 0 \leq i<N_{k} .
$$

We remark that in probabilistic language $Z_{k, N}$ is the conditional expectation of $Y_{k, N}$ under $\mathcal{F}_{k}$. The increasing system of $\sigma$-algebras $\left(\mathcal{F}_{k}\right)_{k}$ forms a so-called filtration, which is the basis for constructing a martingale.

The following lemma describes how good an approximation $Z_{k, N}$ is to $Y_{k, N}$.

Lemma 2.4. For each $1 \leq k \leq N^{9 / 10}$ we have

$$
\left\|Y_{k, N}-Z_{k, N}\right\|_{\infty}=\mathcal{O}\left(\frac{N^{41 / 10}}{A^{N^{1 / 10} / 2}}\right) .
$$

Before we turn to the proof of the lemma, let us quickly reflect on what we are doing here. We have split the sum $\sum_{m<n} G\left(x^{n}-x^{m}\right)$ into block sums $Y_{k, N}$, according to the size of the larger index $n$. We can think of the functions $G\left(x^{n}-x^{m}\right)$ as being quickly oscillating, with the oscillation frequency growing exponentially as a function of $n$. We defined a $\sigma$-field $\mathcal{F}_{k}$, and replaced $Y_{k, N}$ by the conditional expectation of $Y_{k, N}$ under $\mathcal{F}_{k}$. Note that this conditional expectation is a step function, which is $\mathcal{F}_{k}$-measurable. Using the function $\mu_{k}$ from (2.4) we constructed $\mathcal{F}_{k}$ in an "inhomogeneous" way, in the sense that its atoms become finer and finer when moving from $A$ towards $A+1$, which reflects the fact that the "oscillation frequency" of $G\left(x^{n}-x^{m}\right)$ is not everywhere the same, but also increases when $x$ moves from $A$ to $A+1$. We have constructed $\mathcal{F}_{k}$ in such a way that $Z_{k, N}=\mathbb{E}\left(Y_{k, N} \mid \mathcal{F}_{k}\right)$ is a good approximation to $Y_{k, N} \mathbb{1}$ However, since $Z_{k, N}$ is constant on the atoms of $\mathcal{F}_{k}$, we can use the fact that the functions in the next block $Z_{k+2, N}$ oscillate with much higher frequency than those in $Z_{k, N}$, so that they still oscillate quickly on the atoms of $\mathcal{F}_{k}$. Note that indeed the next block is $Z_{k+2, N}$ and not $Z_{k+1, N}$, since we currently only consider odd values of $k$ - this is why we split the whole sum into even and odd parts, to separate the oscillation frequencies in different blocks. So from our construction we essentially have $\mathbb{E}\left(Z_{k+2, N} \mid \mathcal{F}_{k}\right) \approx 0$, and a similar result for any other (odd) index $j>k+2$ instead of $k+2$ (see Lemma 2.5 below). Since by construction $Z_{k, N}$ is $\mathcal{F}_{k}$-measurable, we thus have $\mathbb{E}\left(Z_{k, N} Z_{j, N}\right)=\mathbb{E}\left(\mathbb{E}\left(Z_{k, N} Z_{j, N} \mid \mathcal{F}_{k}\right)\right)=\mathbb{E}\left(Z_{k, N} \mathbb{E}\left(Z_{j, N} \mid \mathcal{F}_{k}\right)\right) \approx 0$. After changing back from $Z$ to $Y$, this is essentially Lemma 2.2. The proof of Lemma 2.3 is obtained in a very similar way.

\footnotetext{
${ }^{1}$ We write $\mathbb{E}$ for the expectation (integral) with respect to $x$, on the interval $[A, A+1$ ) equipped with Borel sets and Lebesgue measure.
} 
Proof of Lemma 2.4. Let $x \in[A, A+1)$. Suppose $0 \leq i<N_{k}$ is such that $x \in\left[z_{i, k}, z_{i+1, k}\right)$. Then it is a consequence of $Y_{k, N}$ being a continuous function that there exists $y \in\left[z_{i, k}, z_{i+1, k}\right]$ such that $Z_{k, N}(x)=Y_{k, N}(y)$. This observation will allow us to use the mean value theorem to bound $\left|Y_{k, N}(x)-Z_{k, N}(x)\right|$. To prove our lemma we will also require the following estimates:

a) For each $1 \leq k \leq N^{9 / 10}$ and $x \in[A, A+1)$ we have

$$
\left|Y_{k, N}^{\prime}(x)\right|=\mathcal{O}\left(N^{41 / 10} \cdot x^{k N^{1 / 10}}\right) .
$$

This bound follows by applying the chain rule to each term in the summation for $Y_{k, N}$ together with the assumption $\left\|F_{N}^{\prime}\right\|_{\infty}=\mathcal{O}\left(N^{2}\right)$.

b) For each $1 \leq k \leq N^{9 / 10}$ we have

$$
\frac{k N^{1 / 10}}{z_{i, k} 2^{\mu_{k}\left(z_{i, k}\right)}}=\mathcal{O}(1)
$$

This follows from the definition of the function $\mu_{k}$.

c) For all $x \in \mathbb{R}$ and $r>0$ we have

$$
(1+x)^{r} \leq e^{r x}
$$

We now apply these bounds together with the mean value theorem:

$$
\begin{aligned}
\left|Y_{k, N}(x)-Z_{k, N}(x)\right| & =\left|Y_{k, N}(x)-Y_{k, N}(y)\right| \\
& \leq|x-y| \sup _{w \in\left[z_{i, k}, z_{i+1, k}\right]}\left|Y_{k, N}^{\prime}(w)\right| \\
& \stackrel{(2.5)}{=} \mathcal{O}\left(2^{-\mu_{k}\left(z_{i, k}\right)} N^{41 / 10}\left(z_{i, k}+2^{-\mu_{k}\left(z_{i, k}\right)}\right)^{k N^{1 / 10}}\right) \\
& =\mathcal{O}\left(2^{-\mu_{k}\left(z_{i, k}\right)} N^{41 / 10} z_{i, k}^{k N^{1 / 10}}\left(1+\frac{1}{z_{i, k} 2^{\mu_{k}\left(z_{i, k}\right)}}\right)^{k N^{1 / 10}}\right) \\
& \stackrel{(2.7)}{=} \mathcal{O}\left(2^{-\mu_{k}\left(z_{i, k}\right)} N^{41 / 10} z_{i, k}^{k N^{1 / 10}} \exp \left(\frac{k N^{1 / 10}}{z_{i, k} 2^{\mu_{k}\left(z_{i, k}\right)}}\right)\right) \\
& \stackrel{(2.6)}{=} \mathcal{O}\left(2^{-\mu_{k}\left(z_{i, k}\right)} N^{41 / 10} z_{i, k}^{k N^{1 / 10}}\right)
\end{aligned}
$$




$$
\begin{aligned}
& =\mathcal{O}\left(\frac{z_{i, k}^{k N^{1 / 10}} N^{41 / 10}}{z_{i, k}^{(k+1 / 2) N^{1 / 10}}}\right) \\
& =\mathcal{O}\left(\frac{N^{41 / 10}}{z_{i, k}^{N^{1 / 10} / 2}}\right) \\
& =\mathcal{O}\left(\frac{N^{41 / 10}}{A^{N^{1 / 10} / 2}}\right) .
\end{aligned}
$$

In the final line we used that $A \leq z_{i, k}$ for all $0 \leq i<N_{k}$ and $1 \leq k \leq N^{9 / 10}$. Since $x$ was arbitrary our result follows.

Lemma 2.5. Let $j, k \in \mathbb{N}$ be such that $k-j \geq 2$ and let $0 \leq i<N_{j}$. Then

$$
\frac{1}{\mathcal{L}\left(\left[z_{i, j}, z_{i+1, j}\right)\right)} \int_{z_{i, j}}^{z_{i+1, j}} Y_{k, N} d x=\mathcal{O}\left(\frac{\log N}{N^{29 / 10}}\right) .
$$

Thus we have

$$
\mathbb{E}\left(Z_{k, N} \mid \mathcal{F}_{j}\right)(x)=\mathbb{E}\left(Y_{k, N} \mid \mathcal{F}_{j}\right)(x)=\mathcal{O}\left(\frac{\log N}{N^{29 / 10}}\right),
$$

uniformly for all $x \in[A, A+1)$.

To prove Lemma 2.5, we will use the van der Corput lemma (see for example [21, p. 15]).

Lemma 2.6 (van der Corput lemma). Let $\phi:[a, b] \rightarrow \mathbb{R}$ be differentiable. Assume that $\phi^{\prime}(x) \geq \gamma$ for all $x \in[a, b]$, and $\phi^{\prime}$ is monotonic on $[a, b]$. Then

$$
\left|\int_{a}^{b} e^{2 \pi i \phi(x)} d x\right| \leq \gamma^{-1}
$$

Proof of Lemma 2.5. We begin by focusing on the first part of our lemma.

Since $G_{N}$ is a differentiable function satisfying $G_{N}(x+1)=G_{N}(x)$ for all $x \in \mathbb{R}$, we know that it equals its Fourier series, i.e.,

$$
G_{N}(x)=\sum_{l \in \mathbb{Z}} c_{l, N} e^{2 \pi i l x} .
$$

Where $c_{0, N}=0$ because $\int_{0}^{1} G_{N} d x=0$. We let

$$
P_{N}:=\sum_{|l| \leq N^{6}} c_{l, N} e^{2 \pi i l x}
$$

and

$$
R_{N}:=G_{N}-P_{N}
$$


By assumption we have $\int_{0}^{1}\left|G_{N}\right| d x=\mathcal{O}(1)$, which directly implies that

$$
\left|c_{l, N}\right|=\mathcal{O}(1), l \neq 0
$$

We also have the bound

$$
\left\|R_{N}\right\|_{\infty}=\mathcal{O}\left(\frac{\log N}{N^{4}}\right) .
$$

The estimate (2.9) is a variant of Jackson's inequality from approximation theory; we have not found a good reference except in Jackson's old book, so for the convenience of the reader we give a derivation of this estimate at the end of the present proof.

By an application of the triangle inequality we obtain

$$
\begin{aligned}
\left|\int_{z_{i, j}}^{z_{i+1, j}} G_{N}\left(x^{n}-x^{m}\right) d x\right| & \leq \sum_{|l| \leq N^{6}}\left|\int_{z_{i, j}}^{z_{i+1, j}} c_{l, N} e^{2 \pi i l\left(x^{n}-x^{m}\right)} d x\right| \\
& +\left|\int_{z_{i, j}}^{z_{i+1, j}} R_{N}\left(x^{n}-x^{m}\right) d x\right| .
\end{aligned}
$$

Applying (2.9) to the second term on the right hand side of (2.10) we have

$$
\left|\int_{z_{i, j}}^{z_{i+1, j}} R_{N}\left(x^{n}-x^{m}\right) d x\right|=\mathcal{O}\left(\frac{\mathcal{L}\left(\left[z_{i, j}, z_{i+1, j}\right]\right) \log N}{N^{4}}\right) .
$$

We now apply the van der Corput lemma to the first term on the right hand side of (2.10). Let $\phi_{l}(x)=l\left(x^{n}-x^{m}\right)$. For any $x \in\left[z_{i, j}, z_{i+1, j}\right]$ we have

$$
\begin{aligned}
\phi_{l}^{\prime}(x) & =l\left(n x^{n-1}-m x^{m-1}\right) \\
& \geq \ln \left(x^{n-1}-x^{m-1}\right) \\
& \geq \ln z_{i, j}^{n-1}\left(1-z_{i, j}^{-1}\right) .
\end{aligned}
$$

One can also easily check that $\phi_{l}^{\prime \prime}(x)=l\left(n(n-1) x^{n-2}-m(m-1) x^{m-2}\right)>0$ for all $x \in\left[z_{i, j}, z_{i+1, j}\right]$, since all real solutions $x$ to the equality $x^{n-m}=m(m-1) /(n(n-1))$ are obviously smaller than 1 (provided that $n \geq 2$ ). Therefore $\phi_{l}^{\prime}$ is monotonic. Applying Lemma 2.6 together with (2.8) we see that

$$
\left|\int_{z_{i, j}}^{z_{i+1, j}} c_{l, N} e^{2 \pi i l\left(x^{n}-x^{m}\right)} d x\right|=\mathcal{O}\left(\frac{1}{\ln z_{i, j}^{n-1}}\right) .
$$


Which implies the following bound for the first term on the right hand side of (2.10):

$$
\sum_{|l| \leq N^{6}}\left|\int_{z_{i, j}}^{z_{i+1, j}} c_{l, N} e^{2 \pi i l\left(x^{n}-x^{m}\right)} d x\right|=\mathcal{O}\left(\frac{\log N}{n z_{i, j}^{n-1}}\right) .
$$

Using (2.11) and (2.12) together with the definition of $\mu_{j}\left(z_{i, j}\right)$, the fact $k-j \geq 2$, and $n \in \Delta_{k, N}$, we obtain:

$$
\begin{aligned}
\left|\frac{1}{\mathcal{L}\left(\left[z_{i, j}, z_{i+1, j}\right)\right)} \int_{z_{i, j}}^{z_{i+1, j}} G_{N}\left(x^{n}-x^{m}\right) d x\right| & =\mathcal{O}\left(\frac{z_{i, j}^{(j+1 / 2) N^{1 / 10}} \log N}{n z_{i, j}^{n-1}}+\frac{\log N}{N^{4}}\right) \\
& =\mathcal{O}\left(\frac{\log N}{n z_{i, j}^{N^{1 / 10} / 2}}+\frac{\log N}{N^{4}}\right) \\
& =\mathcal{O}\left(\frac{\log N}{N^{4}}\right) .
\end{aligned}
$$

In the last line we used that $z_{i, j}^{-N^{1 / 10} / 2} \leq A^{-N^{1 / 10} / 2}$, and this upper bound decays to zero faster than any negative power of $N$. Applying (2.13) to each term in the summation for $Y_{k, N}$, together with the fact that this summation consists of $\mathcal{O}\left(N^{11 / 10}\right)$ terms, we obtain:

$$
\frac{1}{\mathcal{L}\left(\left[z_{i, j}, z_{i+1, j}\right)\right)} \int_{z_{i, j}}^{z_{i+1, j}} Y_{k, N} d x=\mathcal{O}\left(\frac{\log N}{N^{29 / 10}}\right) .
$$

For the second assertion of the lemma, we just note that by construction $\mathcal{F}_{j}$ is a sub $\sigma$-algebra of $\mathcal{F}_{k}$, and consequently $\mathbb{E}\left(Z_{k, N} \mid \mathcal{F}_{j}\right)=\mathbb{E}\left(Y_{k, N} \mid \mathcal{F}_{j}\right)$.

We conclude the proof of Lemma 2.5 by verifying (2.9). Let

$$
D(x)=\frac{\sin \left(2 \pi\left(N^{6}+1 / 2\right) x\right)}{\sin (\pi x)}
$$

be the Dirichlet kernel of order $N^{6}$. We have

$$
\begin{aligned}
R_{N}(x) & =G_{N}(x)-\int_{0}^{1} G_{N}(y) D(x-y) d y \\
& =G_{N}(x)-\int_{0}^{1}\left(G_{N}(x)+\left(G_{N}(x-y)-G_{N}(x)\right)\right) D(y) d y \\
& =-\int_{0}^{1}\left(G_{N}(x-y)-G_{N}(x)\right) D(y) d y
\end{aligned}
$$

since $\int D d x=1$. By periodicity, we consequently have

$$
\left|R_{N}(x)\right| \leq\left|\int_{-1 / N^{6}}^{1 / N^{6}}\left(G_{N}(x-y)-G_{N}(x)\right) D(y) d y\right|
$$


ON THE PAIR CORRELATIONS OF POWERS OF REAL NUMBERS

$$
+\left|\int_{1 / N^{6}}^{1-1 / N^{6}}\left(G_{N}(x-y)-G_{N}(x)\right) D(y) d y\right| .
$$

By assumption the derivative of $G_{N}$ is uniformly bounded by $\mathcal{O}\left(N^{2}\right)$, and thus

$$
\left|\left(G_{N}(x-y)-G_{N}(x)\right) D(y)\right| \leq\left|\frac{G_{N}(x-y)-G_{N}(x)}{\sin \pi y}\right|=\mathcal{O}\left(N^{2}\right) .
$$

Accordingly, we have

$$
\left|\int_{-1 / N^{6}}^{1 / N^{6}}\left(G_{N}(x-y)-G_{N}(x)\right) D(y) d y\right|=\mathcal{O}\left(\frac{1}{N^{4}}\right),
$$

which gives the desired bound for the integral in line (2.14). To estimate the second integral, we use integration by parts and obtain

$$
\begin{aligned}
& \left|\int_{1 / N^{6}}^{1-1 / N^{6}}\left(G_{N}(x-y)-G_{N}(x)\right) D(y) d y\right| \\
= & \frac{1}{2 \pi\left(N^{6}+1 / 2\right)}\left|\int_{1 / N^{6}}^{1-1 / N^{6}}\left(\frac{G_{N}(x-y)-G_{N}(x)}{\sin \pi y}\right)^{\prime} \cos \left(2 \pi\left(N^{6}+1 / 2\right) y\right) d y\right|+\mathcal{O}\left(\frac{1}{N^{4}}\right),
\end{aligned}
$$

where we estimated the contribution coming from the boundary terms using (2.16) . Now we have

$$
\begin{aligned}
\left|\left(\frac{G_{N}(x-y)-G_{N}(x)}{\sin \pi y}\right)^{\prime}\right| & \leq\left|\frac{G_{N}^{\prime}(x-y)}{\sin \pi y}\right|+\pi\left|\frac{G_{N}(x-y)-G_{N}(x)}{(\sin \pi y)^{2}}\right| \\
& =\mathcal{O}\left(\frac{N^{2}}{\sin \pi y}+\frac{N^{2} \min (y, 1-y)}{(\sin \pi y)^{2}}\right) \\
& =\mathcal{O}\left(\frac{N^{2}}{\sin \pi y}\right) .
\end{aligned}
$$

Thus we obtain

$$
\begin{aligned}
\left|\int_{1 / N^{6}}^{1-1 / N^{6}}\left(G_{N}(x-y)-G_{N}(x)\right) D(y) d y\right| & =\mathcal{O}\left(\frac{1}{N^{4}} \int_{1 / N^{6}}^{1-1 / N^{6}} \frac{1}{\sin \pi y} d y\right)+\mathcal{O}\left(\frac{1}{N^{4}}\right) \\
& =\mathcal{O}\left(\frac{\log N}{N^{4}}\right) .
\end{aligned}
$$

This gives the desired bound for the integral on the right-hand side of line (2.15), and thus establishes (2.9) as desired.

With Lemma 2.4 and Lemma 2.5 we can now prove Lemma 2.2. Before giving our proof we recall some well known properties of conditional expectation.

Let $(X, \mathcal{B}, \mu)$ be a probability space. 
- Suppose $F, G: X \rightarrow \mathbb{R}$ are random variables and $\mathcal{H}$ is a sub $\sigma$-algebra of $\mathcal{B}$. If $F$ is $\mathcal{H}$-measurable then $\mathbb{E}(F \cdot G \mid \mathcal{H})=F \cdot \mathbb{E}(G \mid \mathcal{H})$.

- Suppose $F: X \rightarrow \mathbb{R}$ is a random variable and $\mathcal{H}$ is a sub $\sigma$-algebra of $\mathcal{B}$. Then

$$
\int_{X}|\mathbb{E}(F \mid \mathcal{H})| d \mu \leq \int_{X}|F| d \mu .
$$

Proof of Lemma 2.2. Let $k, j \in \mathbb{N}$ be such that $k-j \geq 2$. By the triangle inequality:

$$
\begin{aligned}
& \left|\int_{A}^{A+1} Y_{j, N} \cdot Y_{k, N} d x\right| \\
= & \left|\int_{A}^{A+1} Y_{j, N} \cdot Y_{k, N}-Z_{j, N} \cdot Z_{k, N} d x\right|+\left|\int_{A}^{A+1} Z_{j, N} \cdot Z_{k, N} d x\right| .
\end{aligned}
$$

Using the properties of conditional expectation mentioned above, together with Lemma 2.5, we see that the following holds:

$$
\begin{aligned}
\left|\int_{A}^{A+1} Z_{j, N} \cdot Z_{k, N} d x\right| & =\left|\int_{A}^{A+1} \mathbb{E}\left(Z_{j, N} \cdot Z_{k, N} \mid \mathcal{F}_{j}\right) d x\right| \\
& =\left|\int_{A}^{A+1} Z_{j, N} \cdot \mathbb{E}\left(Z_{k, N} \mid \mathcal{F}_{j}\right) d x\right| \\
& \leq \int_{A}^{A+1}\left|Z_{j, N} \cdot \mathbb{E}\left(Z_{k, N} \mid \mathcal{F}_{j}\right)\right| d x \\
& =\mathcal{O}\left(\frac{\log N}{N^{29 / 10}} \int_{A}^{A+1}\left|Z_{j, N}\right| d x\right) \\
& =\mathcal{O}\left(\frac{\log N}{N^{29 / 10}} \int_{A}^{A+1}\left|Y_{j, N}\right| d x\right) \\
& =\mathcal{O}\left(\frac{\log N}{N^{18 / 10}}\right) .
\end{aligned}
$$

In the final line we used that $\left\|Y_{j, N}\right\|_{\infty}=\mathcal{O}\left(N^{11 / 10}\right)$.

Using Lemma 2.4 and the fact $\left\|Y_{k, N}\right\|_{\infty}=\mathcal{O}\left(N^{11 / 10}\right)$, we similarly obtain:

$$
\begin{aligned}
& \left|\int_{A}^{A+1} Y_{j, N} \cdot Y_{k, N}-Z_{j, N} \cdot Z_{k, N} d x\right| \\
= & \left|\int_{A}^{A+1} Y_{j, N} \cdot Y_{k, N}-\left(Y_{j, N}+\left(Z_{j, N}-Y_{j, N}\right)\right) \cdot\left(Y_{k, N}+\left(Z_{k, N}-Y_{k, N}\right)\right) d x\right| \\
= & \left|\int_{A}^{A+1} Y_{k, N}\left(Z_{j, N}-Y_{j, N}\right)+Y_{j, N}\left(Z_{k, N}-Y_{k, N}\right)+\left(Z_{j, N}-Y_{j, N}\right)\left(Z_{k, N}-Y_{k, N}\right) d x\right| \\
\leq & \int_{A}^{A+1}\left|Y_{k, N}\left(Z_{j, N}-Y_{j, N}\right)\right|+\left|Y_{j, N}\left(Z_{k, N}-Y_{k, N}\right)\right|+\left|\left(Z_{j, N}-Y_{j, N}\right)\left(Z_{k, N}-Y_{k, N}\right)\right| d x
\end{aligned}
$$




$$
=\mathcal{O}\left(\frac{N^{52 / 10}}{A^{N^{1 / 10} / 2}}\right) \text {. }
$$

Substituting this bound as well as (2.18) into (2.17), and using the fact that $A^{-N^{1 / 10} / 2}$ decays to zero faster than any negative power of $N$, we obtain the desired result.

2.2. Proof of Lemma 2.3. We start our proof by choosing $N_{0}$ sufficiently large such that for all $m_{1} \geq N_{0}$, if $n>m_{1}$ and $m_{2}<m_{1}$ then

$$
\left(\left\lfloor A^{m_{1}}-A^{m_{2}}\right\rfloor-2\right)^{1-n / m_{1}} \leq A^{\left(m_{1}-n\right) / 2} .
$$

Applying the triangle inequality for the $L^{2}$ norm twice we obtain:

$$
\begin{aligned}
\left(\int_{A}^{A+1} Y_{k, N}^{2} d x\right)^{1 / 2} & =\left(\int_{A}^{A+1}\left(\sum_{n \in \Delta_{k, N}} \sum_{1 \leq m<n} G_{N}\left(x^{n}-x^{m}\right)\right)^{2} d x\right)^{1 / 2} \\
& \leq \sum_{n \in \Delta_{k, N}}\left(\int_{A}^{A+1}\left(\sum_{1 \leq m<n} G_{N}\left(x^{n}-x^{m}\right)\right)^{2} d x\right)^{1 / 2} \\
& \leq \sum_{n \in \Delta_{k, N}}\left(\int_{A}^{A+1}\left(\sum_{\substack{1 \leq m<n \\
m<N_{0}}} G_{N}\left(x^{n}-x^{m}\right)\right)^{2} d x\right)^{1 / 2} \\
& \left.+\sum_{n \in \Delta_{k, N}}\left(\int_{A}^{A+1}\left(\sum_{\substack{1 \leq m<n \\
m \geq N_{0}}} G_{N}\left(x^{n}-x^{m}\right)\right)^{1 / 2} d x\right)^{2}\right)^{1 / 2} \\
& =\mathcal{O}\left(N^{1 / 10}\right)+\sum_{n \in \Delta_{k, N}}\left(\int_{A}^{A+1}\left(\sum_{\substack{1 \leq m<n \\
m \geq N_{0}}} G_{N}\left(x^{n}-x^{m}\right)\right)^{2}\right.
\end{aligned}
$$

In the final line we used that $\left\|G_{N}\right\|_{\infty}=\mathcal{O}(1)$ and $\# \Delta_{k, N}=N^{1 / 10}$. To complete our proof of Lemma 2.3 we need to obtain good bounds for

$$
\sum_{n \in \Delta_{k, N}}\left(\int_{A}^{A+1}\left(\sum_{\substack{1 \leq m<n \\ m \geq N_{0}}} G_{N}\left(x^{n}-x^{m}\right)\right)^{2} d x\right)^{1 / 2}
$$


Expanding the bracket within the integral we obtain

$$
\sum_{n \in \Delta_{k}, N}\left(\int_{A}^{A+1} \sum_{N_{0} \leq m_{1}, m_{2}<n} G_{N}\left(x^{n}-x^{m_{1}}\right) G_{N}\left(x^{n}-x^{m_{2}}\right) d x\right)^{1 / 2} .
$$

Recall that $G_{N}=F_{N}-\int_{0}^{1} F_{N} d x$. Using this equation, together with the assumptions $0 \leq F_{N} \leq 1$ and $\operatorname{supp}\left(F_{N}\right) \subset \mathbb{Z}+\left[\frac{-2 s}{N}, \frac{2 s}{N}\right]$, we see that for any $N_{0} \leq m_{1}, m_{2}<n$ we have

$$
\begin{aligned}
& \int_{A}^{A+1} G_{N}\left(x^{n}-x^{m_{1}}\right) G_{N}\left(x^{n}-x^{m_{2}}\right) d x \\
= & \int_{A}^{A+1} F_{N}\left(x^{n}-x^{m_{1}}\right) F_{N}\left(x^{n}-x^{m_{2}}\right) d x-\int_{0}^{1} F_{N} d x \int_{A}^{A+1} F_{N}\left(x^{n}-x^{m_{1}}\right) d x \\
& -\int_{0}^{1} F_{N} d x \int_{A}^{A+1} F_{N}\left(x^{n}-x^{m_{2}}\right) d x+\left(\int_{0}^{1} F_{N} d x\right)^{2} \\
\leq & \int_{A}^{A+1} F_{N}\left(x^{n}-x^{m_{1}}\right) F_{N}\left(x^{n}-x^{m_{2}}\right) d x+\mathcal{O}\left(\frac{1}{N^{2}}\right) .
\end{aligned}
$$

To estimate the integral in (2.21), we will make regular use of the following lemma from [5], which we have rewritten slightly to suit our purposes.

Lemma 2.7. [5, Lemma 2.1.] Let $f:[a, b] \rightarrow \mathbb{R}$ be a strictly increasing differentiable convex function and $s>0$. Then for $N$ sufficiently large

$$
\mathcal{L}\left(\alpha \in[a, b]: f(\alpha) \in \mathbb{Z}+\left[\frac{-s}{N}, \frac{s}{N}\right]\right) \leq \frac{4 s(b-a)}{N}+\mathcal{O}\left(\frac{4 s}{N f^{\prime}(a)}\right) .
$$

Lemma 2.8. Let $n>m$, then

$$
\int_{A}^{A+1} F_{N}\left(x^{n}-x^{m}\right)^{2} d x=\mathcal{O}\left(\frac{1}{N}\right)
$$

Proof. Recall that $0 \leq F_{N} \leq 1$ and $\operatorname{supp}\left(F_{N}\right) \subset \mathbb{Z}+\left[\frac{-2 s}{N}, \frac{2 s}{N}\right]$. Therefore $F_{N}^{2}$ can be bounded above by the function $\chi_{\left[\frac{-2 s}{N}, \frac{2 s}{N}\right]}$. Therefore to prove our statement it suffices to show that

$$
\int_{A}^{A+1} \chi_{\left[\frac{-2 s}{N}, \frac{2 s}{N}\right]}\left(x^{n}-x^{m}\right) d x=\mathcal{O}\left(\frac{1}{N}\right) .
$$

But this follows immediately from Lemma 2.7.

The proof of the following lemma uses ideas from [5].

Lemma 2.9. Let $n>m_{1}>m_{2}$ and $m_{1} \geq N_{0}$, then

$$
\int_{A}^{A+1} F_{N}\left(x^{n}-x^{m_{1}}\right) F_{N}\left(x^{n}-x^{m_{2}}\right) d x=\mathcal{O}\left(\frac{1}{N^{2}}+\frac{m_{1} A^{\left(m_{1}-n\right) / 2}}{N n\left(n-m_{1}\right)}\right)
$$


Proof. Since $F_{N}$ is positive and bounded above by $\chi_{\left[\frac{-2 s}{N}, \frac{2 s}{N}\right]}(x)$, it suffices to show that

$$
\int_{A}^{A+1} \chi_{\left[\frac{-2 s}{N}, \frac{2 s}{N}\right]}\left(x^{n}-x^{m_{1}}\right) \chi_{\left[\frac{-2 s}{N}, \frac{2 s}{N}\right]}\left(x^{n}-x^{m_{2}}\right) d x=\mathcal{O}\left(\frac{1}{N^{2}}+\frac{m_{1} A^{\left(m_{1}-n\right) / 2}}{N n\left(n-m_{1}\right)}\right) .
$$

Importantly

$$
\chi_{\left[\frac{-2 s}{N}, \frac{2 s}{N}\right]}\left(x^{n}-x^{m_{1}}\right) \chi_{\left[\frac{-2 s}{N}, \frac{2 s}{N}\right]}\left(x^{n}-x^{m_{2}}\right)=1 \Longrightarrow \chi_{\left[\frac{-4 s}{N}, \frac{4 s}{N}\right]}\left(x^{m_{1}}-x^{m_{2}}\right)=1 .
$$

Therefore

$$
\begin{aligned}
& \int_{A}^{A+1} \chi_{\left[\frac{-2 s}{N}, \frac{2 s}{N}\right]}\left(x^{n}-x^{m_{1}}\right) \chi_{\left[\frac{-2 s}{N}, \frac{2 s}{N}\right]}\left(x^{n}-x^{m_{2}}\right) d x \\
\leq & \int_{A}^{A+1} \chi_{\left[\frac{-2 s}{N}, \frac{2 s}{N}\right]}\left(x^{n}-x^{m_{1}}\right) \chi_{\left[\frac{-4 s}{N}, \frac{4 s}{N}\right]}\left(x^{m_{1}}-x^{m_{2}}\right) d x .
\end{aligned}
$$

It therefore suffices to show that

$$
\int_{A}^{A+1} \chi_{\left[\frac{-2 s}{N}, \frac{2 s}{N}\right]}\left(x^{n}-x^{m_{1}}\right) \chi_{\left[\frac{-4 s}{N}, \frac{4 s}{N}\right]}\left(x^{m_{1}}-x^{m_{2}}\right) d x=\mathcal{O}\left(\frac{1}{N^{2}}+\frac{m_{1} A^{\left(m_{1}-n\right) / 2}}{N n\left(n-m_{1}\right)}\right) .
$$

To each $\left\lfloor A^{m_{1}}-A^{m_{2}}\right\rfloor \leq M \leq\left\lceil(A+1)^{m_{1}}-(A+1)^{m_{2}}\right\rceil$ we let

$$
I_{M}:=\left\{x \in[A, A+1): x^{m_{1}}-x^{m_{2}} \in\left[M-\frac{4 s}{N}, M+\frac{4 s}{N}\right]\right\} .
$$

Importantly each $I_{M}$ is an interval and

$$
\left\{x \in[A, A+1): x^{m_{1}}-x^{m_{2}} \in \mathbb{Z}+\left[-\frac{4 s}{N}, \frac{4 s}{N}\right]\right\}=\bigcup_{M=\left\lfloor A^{m_{1}}-A^{m_{2}}\right\rfloor}^{\left\lceil(A+1)^{m_{1}}-(A+1)^{m_{2}}\right\rceil} I_{M} .
$$

Note that if $M \neq M^{\prime}$ then $I_{M} \cap I_{M^{\prime}}$ is either empty or a single endpoint. Therefore

$$
\begin{aligned}
& \int_{A}^{A+1} \chi_{\left[\frac{-2 s}{N}, \frac{2 s}{N}\right]}\left(x^{n}-x^{m_{1}}\right) \chi_{\left[\frac{-4 s}{N}, \frac{4 s}{N}\right]}\left(x^{m_{1}}-x^{m_{2}}\right) d x \\
= & \sum_{M=\left\lfloor A^{m_{1}}-A^{m_{2}}\right\rfloor}^{\left\lceil(A+1)^{m_{1}}-(A+1)^{m_{2}}\right\rceil} \int_{I_{M}} \chi_{\left[\frac{-2 s}{N}, \frac{2 s}{N}\right]}\left(x^{n}-x^{m_{1}}\right) d x .
\end{aligned}
$$

We let $C_{M}$ denote the left endpoint of $I_{M}$. Applying Lemma 2.7 to each $I_{M}$ we obtain

$$
\begin{aligned}
\sum_{M=\left\lfloor A^{m_{1}}-A^{m_{2}}\right\rfloor}^{\left\lceil(A+1)^{m_{1}}-(A+1)^{m_{2}}\right\rceil} \int_{I_{M}} \chi_{\left[\frac{-2 s}{N}, \frac{2 s}{N}\right]}\left(x^{n}-x^{m_{1}}\right) d x & =\mathcal{O}\left(\sum_{M=\left\lfloor A^{m_{1}}-A^{m_{2}}\right\rfloor}^{\left\lceil(A+1)^{m_{1}}-(A+1)^{m_{2}}\right\rceil} \frac{\mathcal{L}\left(I_{M}\right)}{N}\right) \\
& +\mathcal{O}\left(\sum_{M=\left\lfloor A^{m_{1}}-A^{m_{2}}\right\rfloor}^{\left\lceil(A+1)^{m_{1}}-(A+1)^{m_{2}}\right\rceil} \frac{1}{N n C_{M}^{n}}\right) .
\end{aligned}
$$


We focus on each term on the right hand side of (2.24) individually. Starting with the second term, a simple analysis yields $C_{M} \geq(M-1)^{1 / m_{1}}$. Therefore

$$
\begin{aligned}
\sum_{M=\left\lfloor A^{\left.m_{1}-A^{m_{2}}\right\rfloor}\right.}^{\left\lceil(A+1)^{m_{1}}-(A+1)^{m_{2}}\right\rceil} \frac{1}{N n C_{M}^{n}} & \leq \sum_{M=\left\lfloor A^{m_{1}}-A^{m_{2}}\right\rfloor}^{\left\lceil(A+1)^{m_{1}}-(A+1)^{m_{2}}\right\rceil} \frac{1}{N n(M-1)^{n / m_{1}}} \\
& \leq \int_{\left\lfloor A^{m_{1}}-A^{m_{2}}\right\rfloor}^{\left\lceil(A+1)^{m_{1}}-(A+1)^{m_{2}}\right\rceil+1} \frac{1}{N n(x-2)^{n / m_{1}}} d x \\
& =\frac{1}{N n}\left[\frac{(x-2)^{1-n / m_{1}}}{1-n / m_{1}}\right]_{\left\lfloor\left(A^{m_{1}}-A^{m_{2}}\right\rfloor\right.}^{\left\lceil(A+1)^{m_{1}}-(A+1)^{m_{2}}\right\rceil+1} \\
& \leq \frac{m_{1}\left(\left\lfloor A^{m_{1}}-A^{m_{2}}\right\rfloor-2\right)^{1-n / m_{1}}}{N n\left(n-m_{1}\right)} \\
& \leq \frac{m_{1} A^{\left(m_{1}-n\right) / 2}}{N n\left(n-m_{1}\right)}
\end{aligned}
$$

In the last line we used our assumption $m_{1} \geq N_{0}$.

We now focus on the first term on the right hand side of (2.24). Applying (2.23) and Lemma 2.7 we obtain

$$
\begin{aligned}
\sum_{M=\left\lfloor A^{m_{1}}-A^{m_{2}}\right\rfloor}^{\left\lceil(A+1)^{m_{1}}-(A+1)^{m_{2}}\right\rceil} \frac{\mathcal{L}\left(I_{M}\right)}{N} & =\frac{\mathcal{L}\left(\left\{x \in[A, A+1): x^{m_{1}}-x^{m_{2}} \in \mathbb{Z}+\left[\frac{-4 s}{N}, \frac{4 s}{N}\right]\right\}\right)}{N} \\
& =\frac{1}{N}\left(\mathcal{O}\left(\frac{1}{N}\right)+\mathcal{O}\left(\frac{1}{N m_{1} A^{m_{1}}}\right)\right) \\
& =\mathcal{O}\left(\frac{1}{N^{2}}\right) .
\end{aligned}
$$

Substituting (2.25) and (2.26) into (2.24) we deduce that (2.22) holds. This completes the proof of Lemma 2.9.

Proof of Lemma 2.3. By (2.20) and (2.21) we have

$$
\begin{aligned}
& \left(\int_{A}^{A+1} Y_{k, N}^{2} d x\right)^{1 / 2} \\
\leq & \sum_{n \in \Delta_{k, N}}\left(\int_{A}^{A+1} \sum_{N_{0} \leq m_{1}, m_{2}<n}\left(F_{N}\left(x^{n}-x^{m_{1}}\right) F_{N}\left(x^{n}-x^{m_{2}}\right) d x+\mathcal{O}\left(\frac{1}{N^{2}}\right)\right)\right)^{1 / 2} \\
& +\mathcal{O}\left(N^{1 / 10}\right) .
\end{aligned}
$$


Using Lemma 2.8 and Lemma 2.9 we obtain

$$
\begin{aligned}
& \left(\int_{A}^{A+1} Y_{k, N}^{2} d x\right)^{1 / 2} \\
\leq & \sum_{n \in \Delta_{k, N}}\left(\sum_{N_{0} \leq m_{2}<m_{1}<n} \mathcal{O}\left(\frac{1}{N^{2}}+\frac{m_{1} A^{\left(m_{1}-n\right) / 2}}{N n\left(n-m_{1}\right)}\right)+\sum_{m=N_{0}}^{n} \mathcal{O}\left(\frac{1}{N}\right)\right)^{1 / 2} \\
& +\mathcal{O}\left(N^{1 / 10}\right)
\end{aligned}
$$

Note that

$$
\sum_{m=N_{0}}^{n} \frac{1}{N} \leq 1 \text { and } \sum_{N_{0} \leq m_{2}<m_{1}<n} \frac{1}{N^{2}} \leq 1,
$$

since $n \leq N$. Furthermore, we observe that

$$
\begin{aligned}
\sum_{N_{0} \leq m_{2}<m_{1}<n} \frac{m_{1} A^{\left(m_{1}-n\right) / 2}}{N n\left(n-m_{1}\right)} & =\sum_{m_{2}=N_{0}}^{n-2} \sum_{m_{1}=m_{2}+1}^{n-1} \frac{m_{1} A^{\left(m_{1}-n\right) / 2}}{N n\left(n-m_{1}\right)} \\
& \leq \sum_{m_{2}=N_{0}}^{n-2} \frac{1}{N} \sum_{m_{1}=m_{2}+1}^{n-1} \frac{A^{\left(m_{1}-n\right) / 2}}{\left(n-m_{1}\right)} \\
& \leq \sum_{m_{2}=N_{0}}^{n-2} \frac{1}{N\left(A^{1 / 2}-1\right)} \\
& =\mathcal{O}(1) .
\end{aligned}
$$

In the penultimate inequality we used properties of geometric series, and in the final line we used that $n \leq N$. We have shown that the sum of the terms in the bracket in (2.27) is $\mathcal{O}(1)$. Finally, using this bound together with $\# \Delta_{k, n}=N^{1 / 10}$ we arrive at

$$
\left(\int_{0}^{1} Y_{k, N}^{2} d x\right)^{1 / 2}=\mathcal{O}\left(N^{1 / 10}\right)
$$

which proves Lemma 2.3.

2.3. Proof of Theorem 1.4. Throughout this proof we will use primed summation signs, such as $\sum_{k}^{\prime}$, to indicate that the summation is restricted such that it only contains odd values of $k$. By Markov's inequality and Proposition 2.1 we have

$$
\mathcal{L}\left(x \in[A, A+1):\left|\sum_{1 \leq k \leq N^{9 / 10}}^{\prime} Y_{k, N}\right| \geq N^{6 / 10}\right)
$$




$$
\begin{aligned}
& =\mathcal{L}\left(x \in[A, A+1):\left(\sum_{1 \leq k \leq N^{9 / 10}}^{\prime} Y_{k, N}\right)^{2} \geq N^{12 / 10}\right) \\
& =\mathcal{O}\left(\frac{1}{N^{1 / 10}}\right) .
\end{aligned}
$$

Restricting to 20th powers this bound implies

$$
\mathcal{L}\left(x \in[A, A+1):\left|\sum_{1 \leq k \leq N^{18}}^{\prime} Y_{k, N^{20}}\right| \geq N^{12}\right)=\mathcal{O}\left(\frac{1}{N^{2}}\right)
$$

Clearly

$$
\sum_{N=1}^{\infty} \frac{1}{N^{2}}<\infty
$$

Therefore by the Borel-Cantelli lemma it follows that for Lebesgue almost every $x \in$ $[A, A+1)$ the inequality

$$
\left|\sum_{1 \leq k \leq N^{18}}^{\prime} Y_{k, N^{20}}\right| \geq N^{12}
$$

holds for at most finitely many $N$. Therefore Lebesgue almost every $x \in[A, A+1)$ satisfies

$$
\lim _{N \rightarrow \infty} \frac{\sum_{1 \leq k \leq N^{18}}^{\prime} Y_{k, N^{20}}(x)}{N^{20}}=0
$$

An analogous result holds if the summation is extended over all even, instead of all odd, values of $k$ in the specified range. It follows from the definition of $Y_{k, N}$ that for Lebesgue almost every $x \in[A, A+1)$ we have

$$
\lim _{N \rightarrow \infty} \frac{\sum_{1 \leq m \neq n \leq N^{20}} G_{N^{20}}\left(x^{n}-x^{m}\right)}{N^{20}}=0 .
$$

Recall that $G_{N}=F_{N}-\int_{0}^{1} F_{N} d x$ and $\int_{0}^{1} F_{N} d x=\frac{2 s}{N}+\mathcal{O}\left(N^{-2}\right)$. Using this information in the equation above, we deduce that for Lebesgue almost every $x \in[A, A+1)$ we have

$$
\lim _{N \rightarrow \infty} \frac{\sum_{1 \leq m \neq n \leq N^{20}} F_{N^{20}}\left(x^{n}-x^{m}\right)}{N^{20}}=2 s .
$$

This proves Theorem 1.4. 


\section{Proof of Theorem 1.2}

With Theorem 1.4 we can now prove Theorem 1.2. Let $s>0$ be arbitrary. We can define two sequences of differentiable functions $\left(F_{N}^{1}\right)_{N=1}^{\infty}$ and $\left(F_{N}^{2}\right)_{N=1}^{\infty}$ satisfying the hypotheses of Theorem 1.4, which also satisfy $F_{N}^{1}(x) \leq \chi_{\left[\frac{-s}{N}, \frac{s}{N}\right]}(x) \leq F_{N}^{2}(x)$ for all $x \in \mathbb{R}$ and $N \in \mathbb{N}$. Theorem 1.4 therefore implies that Lebesgue almost every $x \in[A, A+1)$ satisfies

$$
\lim _{N \rightarrow \infty} \frac{\sum_{1 \leq m \neq n \leq N^{20}} \chi_{\left[\frac{-s}{N^{20}}, \frac{s}{\left.N^{20}\right]}\right.}\left(x^{n}-x^{m}\right)}{N^{20}}=2 s .
$$

Let $S \subset(0, \infty)$ be a countable and dense subset. Since the parameter $s$ above was arbitrary, for Lebesgue almost every $x \in[A, A+1$ ), equation (3.1) indeed holds for all $s \in S$. Using the density of $S$ and an approximation argument, it follows that for Lebesgue almost every $x \in[A, A+1)(\underline{3.1})$ holds for all $s \in(0, \infty)$.

Now we show how to remove the restriction to 20 th powers. To any $N \in \mathbb{N}$ we associate the quantity $M_{N} \in \mathbb{N}$ defined via the inequalities

$$
M_{N}^{20} \leq N<\left(M_{N}+1\right)^{20}
$$

Observe that

$$
\frac{\left(M_{N}+1\right)^{20}}{M_{N}^{20}} \rightarrow 1 \quad \text { as } N \rightarrow \infty .
$$

Let $\epsilon>0$ be arbitrary. From (3.1) we deduce that for Lebesgue almost every $x \in[A, A+1$, for every $s>0$, we have

$$
\begin{aligned}
& \limsup _{N \rightarrow \infty} \frac{\sum_{1 \leq m \neq n \leq N} \chi_{\left[\frac{-s}{N}, \frac{s}{N}\right]}\left(x^{n}-x^{m}\right)}{N} \\
\leq & \limsup _{N \rightarrow \infty} \frac{\sum_{1 \leq m \neq n \leq\left(M_{N}+1\right)^{20}} \chi_{\left[\frac{-s-\epsilon}{\left(M_{N}+1\right)^{20}}, \frac{s+\epsilon}{\left(M_{N}+1\right)^{20}}\right]}\left(x^{n}-x^{m}\right)}{M_{N}^{20}} \\
= & \limsup _{N \rightarrow \infty} \frac{\left(M_{N}+1\right)^{20}}{M_{N}^{20}} \frac{\sum_{1 \leq m \neq n \leq\left(M_{N}+1\right)^{20}} \chi_{\left[\frac{-s-\epsilon}{\left(M_{N}+1\right)^{20}}, \frac{s+\epsilon}{\left(N_{M}+1\right)^{20}}\right]}\left(x^{n}-x^{m}\right)}{\left(M_{N}+1\right)^{20}} \\
= & 2(s+\epsilon) .
\end{aligned}
$$

Since $\epsilon$ was arbitrary, we see that for Lebesgue almost every $x \in[A, A+1)$, for every $s>0$ we have

$$
\limsup _{N \rightarrow \infty} \frac{\sum_{1 \leq m \neq n \leq N} \chi_{\left[\frac{-s}{N}, \frac{s}{N}\right]}\left(x^{n}-x^{m}\right)}{N} \leq 2 s
$$


The corresponding lower bound can be obtained analogously. Therefore, for Lebesgue almost every $x \in[A, A+1)$, for every $s>0$ we have

$$
\lim _{N \rightarrow \infty} \frac{\sum_{1 \leq m \neq n \leq N} \chi_{\left[\frac{-s}{N}, \frac{s}{N}\right]}\left(x^{n}-x^{m}\right)}{N}=2 s .
$$

Since $A>1$ was arbitrary, we see that for Lebesgue almost every $x>1$, for every $s>0$ we have

$$
\lim _{N \rightarrow \infty} \frac{\sum_{1 \leq m \neq n \leq N} \chi_{\left[\frac{-s}{N}, \frac{s}{N}\right]}\left(x^{n}-x^{m}\right)}{N}=2 s .
$$

This completes the proof of Theorem 1.2.

Acknowledgements. The first author is supported by the Austrian Science Fund (FWF), projects F-5512, I-3466 and Y-901.

\section{REFERENCES}

[1] C. Aistleitner, Quantitative uniform distribution results for geometric progressions, Israel J. Math. 204 (2014), no. 1, 155-197.

[2] C. Aistleitner, T. Lachmann, F. Pausinger, Pair correlations and equidistribution, J. Number Th. 182 (2018), 206-220.

[3] C. Aistleitner, G. Larcher, M. Lewko, Additive energy and the Hausdorff dimension of the exceptional set in metric pair correlation problems, with an appendix by Jean Bourgain, Israel J. Math. 222 (2017), no. $1,463-485$.

[4] S. Baker, Equidistribution results for self-similar measures, arXiv:2002.11607.

[5] S. Baker, Equidistribution results for sequences of polynomials, J. Number Theory, to appear.

[6] S. Baker, On the distribution of powers of real numbers modulo 1. Unif. Distrib. Theory 10 (2015), no. $2,67-75$.

[7] I. Berkes. On the asymptotic behaviour of $S f\left(n_{k} x\right)$. Main theorems. Z. Wahrscheinlichkeitstheorie und Verw. Gebiete 34 (1976), no. 4, 319-345.

[8] I.Berkes. On the asymptotic behaviour of $S f\left(n_{k} x\right)$. Applications. Z. Wahrscheinlichkeitstheorie und Verw. Gebiete 34 (1976), no. 4, 347-365.

[9] I. Berkes, W. Philipp, R.F. Tichy, Pair correlations and U-statistics for independent and weakly dependent random variables, Illinois J. Math. 45 (2001), no. 2, 559-580.

[10] T.F. Bloom, S. Chow, A. Gafni, A. Walker, Additive energy and the metric Poissonian property, Mathematika, 64(3), 679-700.

[11] Y. Bugeaud, Distribution modulo one and Diophantine approximation, Cambridge Tracts in Mathematics, 193. Cambridge University Press, Cambridge, 2012.

[12] Y. Bugeaud, L. Liao, M. Rams, Metrical results on the distribution of fractional parts of powers of real numbers. Proc. Edinburgh Math. Soc. 62 (2019), 505-521

[13] Y. Bugeaud, V. Moshchevitin, On fractional parts of powers of real numbers close to 1, Math. Z. 271 (2012), no. 3-4, 627-637. 
[14] A. Dubickas, On the powers of some transcendental numbers, Bull. Austral. Math. Soc. 76 (2007), no. 3, 433-440.

[15] S. Grepstad, G. Larcher, On pair correlation and discrepancy, Arch. Math. 109 (2017), no. 2, $143-149$.

[16] G. H. Hardy, A problem of Diophantine approximation, J. Indian Math. Soc. 11 (1919), $162-166$.

[17] D.R. Heath-Brown, Pair correlation for fractional parts of $\alpha n^{2}$, Math. Proc. Cambridge Philos. Soc. 148 (2010), no. 3, 385-407.

[18] J.-P. Kahane, Sur la répartition des puissances modulo 1, C. R. Math. Acad. Sci. Paris 352 (2014), no. 5, 383-385.

[19] J. F. Koksma, Ein mengentheoretischer Satz über die Gleichverteilung modulo Eins, Compositio Math. 2 (1935), 250-258.

[20] J. F. Koksma, Sur la théorie métrique des approximations diophantiques, Indagationes Math. 7, 54-70 (1945).

[21] L. Kuipers, H. Niederreiter, Uniform distribution of sequences, Wiley-Interscience, John Wiley \& Sons, New York-London-Sydney, 1974.

[22] G. Larcher, W. Stockinger, Some negative results related to Poissonian pair correlation problems, Discrete Math., to appear.

[23] J. Marklof, The Berry-Tabor Conjecture, In: Casacuberta C., Miro-Roig R.M., Verdera J., XamboDescamps S. (eds), European Congress of Mathematics. Progress in Mathematics, vol 202. Birkhäuser, Basel, 2001.

[24] H. Niederreiter, R.F. Tichy, Solution of a problem of Knuth on complete uniform distribution of sequences, Mathematika 32 (1985), no. 1, 26-32.

[25] W. Philipp, W. Stout, Almost sure invariance principles for partial sums of weakly dependent random variables. Mem. Amer. Math. Soc. 2 (1975), issue 2, no. 161.

[26] C. Pisot, La répartition modulo 1 et les nombres algébriques, Ann. Scuola Norm. Sup. Pisa Cl. Sci. (2) 7 (1938), no. 3-4, 205-248.

[27] C. Pisot, Sur la répartition modulo 1 des puissances successives d'un même nombre, C.R. Acad. Sci. Paris 204 (1937), 312-314.

[28] Z. Rudnick, P. Sarnak, The pair correlation function of fractional parts of polynomials, Comm. Math. Phys. 194 (1998), no. 1, 61-70.

[29] Z. Rudnick, P. Sarnak, A. Zaharescu, The distribution of spacings between the fractional parts of $n^{2} \alpha$, Invent. Math. 145 (2001), no. 1, 37-57.

[30] Z. Rudnick, A. Zaharescu, A metric result on the pair correlation of fractional parts of sequences, Acta Arith. 89 (1999), no. 3, 283-293.

[31] Z. Rudnick, A. Zaharescu, The distribution of spacings between fractional parts of lacunary sequences, Forum Math. 14 (2002), no. 5, 691-712.

[32] J. Truelsen, Divisor problems and the pair correlation for the fractional parts of $n^{2} \alpha$, Int. Math. Res. Not. IMRN 2010, no. 16, 3144-3183. 
Christoph Aistleitner: Graz University of Technology, Institute of Analysis and NumBer Theory, Steyrergasse 30/II, 8010 Graz, Austria

E-mail address: aistleitner@math.tugraz.at

Simon Baker: School of Mathematics, University of Birmingham, Birmingham, B15 2Tt, UK

E-mail address: simonbaker412@gmail.com 